\title{
lonospheric disturbance caused by artificial plasma clouds under different release conditions
}

\author{
Xiaoli Zhu®, Yaogai Hu*, Zhengyu Zhao, Binbin Ni and Yuannong Zhang
}

\begin{abstract}
The generation and evolution of artificial plasma clouds is a complicated process that is strongly dependent on the background environment and release conditions. In this paper, based on a three-dimensional two-species fluid model, the evolution characteristics of artificial plasma clouds under various release conditions were analyzed numerically. In particular, the effect of ionospheric density gradient and ambient horizontal wind field was taken into account in our simulation. The results show that an asymmetric plasma cloud structure occurs in the vertical direction when a nonuniform ionosphere is assumed. The density, volume, and expansion velocity of the artificial plasma cloud vary with the release altitude, mass, and initial ionization rate. The initial release velocity can change the cloud's movement and overall distribution. With an initial velocity perpendicular to the magnetic field, an $\mathrm{O}^{+}$density cavity and two bumps exist. When there is an initial velocity parallel to the magnetic field, the generated plasma cloud is bulb-shaped, and only one $\mathrm{O}^{+}$density cavity and one density bump are created. Compared to the cesium case, barium clouds expand more rapidly. Moreover, $\mathrm{Cs}^{+}$clouds have a higher density than $\mathrm{Ba}^{+}$clouds, and the snowplow effect of $\mathrm{Cs}^{+}$is also stronger.
\end{abstract}

Keywords: Artificial plasma cloud, Chemical release, lonosphere, Electron density disturbance, Three-dimensional fluid model

\section{Introduction}

As early as the 1960s, the disturbance of electron density in the ionosphere during the rocket launching process was first discovered. Due to its advantages of low ionization potential, easy gasification, and easy observation, barium $(\mathrm{Ba})$ has become the most commonly used substance in space experiments (e.g., Foppl et al. 1967; Rosenberg 1971; Haerendel et al. 1967; Valenzuela et al. 1986; Huba et al. 1992). In recent years, lanthanide metals such as samarium have also been used in active space experiments because their ionization is independent of sunlight (Zhao et al. 2016; Caton et al. 2017). In summary, artificially created plasma clouds by the release of chemical substances in the ionosphere have important

\footnotetext{
*Correspondence: yaogaihu@whu.edu.cn

Department of Electronic Information, Wuhan University, Wuchang District, Wuhan 430000, China
}

applications in studying related space physics problems, measuring wind fields and electromagnetic fields in the upper atmosphere, artificially modifying the ionosphere, and affecting the short-wave communication and satellite communication. (Pavlov et al. 1993; Oraevsky et al. 2002; Xie et al. 2015).

The main processes of the generation and evolution of artificial plasma clouds in the ionosphere include the expansion of neutral clouds, the photoionization process and the movement of charged particles bound by magnetic fields. The evolution characteristics of artificial plasma clouds have been studied for many years both experimentally and theoretically (e.g., Lloyd and Haerendel 1973; Morse and Destler 1973; Mitchell et al. 1985; Bernhardt et al. 1987; Zakharov 2002; Xie et al. 2014). In 1967, Haerendel et al. conducted a preliminary qualitative discussion on artificial plasma clouds through a simplified low-density perturbation model. Subsequently, 
some one-dimensional, idealized cloud models were used to study the diffusion and classical effect of artificial plasma clouds (e.g., Scholer 1970; Samir et al. 1983; Schunk and Szuszczewicz 1988, 1991). Mitchell et al. (1985) presented a two-dimensional, electrostatic model to simulate a plasma cloud injected transverse to the ambient geomagnetic field with high velocities. To comprehensively describe the expansion and three-dimensional motion of artificial plasma clouds, more detailed three-dimensional models have been established (e.g., Rozhansky et al. 1990; Drake et al. 1988; Zalesak et al. 1988, 1990; Gatsonis and Hastings 1991; Ma and Schunk 1991, 1993, 1994; Delamere et al. 2001; Xie et al. 2014), and the expansion characteristics of plasma clouds under the influence of background neutral wind, electromagnetic fields, collisions between particles and inertia have been studied.

Previous studies have indicated that artificial plasma cloud evolution is complicated and strongly depends on the background environment and release conditions. In 1990, a two-dimensional fluid model was used to study the motion of artificial plasma clouds under various geophysical and release conditions, such as the solar cycle, geomagnetic activity, release altitudes and injection velocities. (Ma and Schunk 1990). After that, Ma and Schunk $(1991,1993)$ explored the effects of variable neutral wind, cloud sizes, electron temperature, and release velocities on short-term $(\sim 10 \mathrm{~s})$ plasma cloud expansion based on a three-dimensional model. Although significant progress has been made in understanding the effects of release conditions on the evolution of plasma clouds, most of those models assumed a uniform background ionosphere and concentrated on relatively short time scales. In this paper, the background density gradient, temperature gradient, and ambient horizontal wind field were taken into account in our simulation, and the longterm distribution of cloud expansion can be predicted through this model.

The photoionization of barium in the ionosphere relies on sunlight. As the element with the lowest ionization potential in the alkali metal group, cesium (Cs) can also be ionized through thermal ionization in addition to photoionization, breaking the restriction of light on experimental conditions. Therefore, cesium was used as the release substance in early active space experiments (Pressman et al. 1960; Holmgren et al. 1981; Eliason et al. 1988). Compared with studies of barium, simulation and experimental studies of cesium release are rarely reported and not sufficient. In general, it is of great significance for the understanding and exploration of the Earth's space environment to transform and utilize the space environment with the release of chemical substances. Carrying out the comparative simulation of the evolution characteristics of released chemicals under different release conditions, and exploring the influence of different released substances on ionospheric disturbance are significant in guiding the selection of the release substances and conditions in active space experiments.

In this paper, based on a three-dimensional twospecies fluid model, the evolution characteristics of artificial plasma clouds under various release conditions were studied through quantitative numerical simulations. The effects of the release altitude $(220 \mathrm{~km}$ and $300 \mathrm{~km})$, species (Ba and Cs), mass $(1 \mathrm{~kg}$, $10 \mathrm{~kg}$ and $100 \mathrm{~kg})$, initial ionization probability $(0 \%$, $20 \%$ and $80 \%$ ) and release velocity (parallel and perpendicular to the magnetic field) were systematically analyzed.

\section{Model description Diffusion of charged particles}

Due to the different spatial distributions between barium ions $\left(\mathrm{Ba}^{+}\right)$and background ions, the different ions are treated separately. In addition, oxygen ions $\left(\mathrm{O}^{+}\right)$ are the dominant ion component of the ionospheric $\mathrm{F}$ region, so we considered a two-species model of $\mathrm{Ba}^{+}$ and $\mathrm{O}^{+}$. Ignoring the density change in background oxygen ions caused by chemical reactions and assuming that the generation of ion clouds is only from photoionization, the density of charged particles is controlled by the continuity equation:

$$
\frac{\partial n_{p}}{\partial t}=-\nabla \cdot\left(n_{p} \boldsymbol{u}_{p}\right)+P_{p}-L_{p}
$$

where the subscript $p$ represents $\mathrm{Ba}^{+}$or $\mathrm{O}^{+}, n_{p}$ and $\boldsymbol{u}_{p}$ represent the number density and drift velocity, respectively. $\nabla \bullet\left(n_{p} \boldsymbol{u}_{p}\right)$ is the convection term. $P_{p}$ and $L_{p}$ represent the generation term and loss term, respectively. For $\mathrm{O}^{+}$, the density is assumed to be constant, that is, the generation term and loss term are set to be zero. For $\mathrm{Ba}^{+}, P_{B a^{+}}=\sigma n_{s}$, where $\sigma=0.0357 s^{-1}$ is the photoionization generation rate of the ion cloud (Mitchell et al. 1985), and $n_{s}$ is the number density of the released neutral atoms. It is assumed that the photoionization generation rate is the net generation rate of $\mathrm{Ba}^{+}$, that is, the charge exchange reaction $\left(\mathrm{Ba}+\mathrm{O}^{+} \rightarrow \mathrm{Ba}^{+}+\mathrm{O}\right)$ and recombination reaction $\left(\mathrm{Ba}^{+}+e^{-} \rightarrow \mathrm{Ba}+h v\right)$ are ignored, so $L_{B a^{+}}=0$. According to Eq. (1), as long as the drift velocities of $\mathrm{Ba}^{+}$and $\mathrm{O}^{+}$and the number density of neutral barium clouds are calculated, the number density of plasma clouds varies with time and space can be obtained.

The velocity of charged particles is calculated by the momentum equation: 


$$
n_{p} m_{p} \frac{d \boldsymbol{u}_{p}}{d t}+\nabla p_{p}-n_{p} q_{p}\left(\boldsymbol{E}+\boldsymbol{u}_{p} \times \boldsymbol{B}\right)=\frac{\delta \boldsymbol{M}_{p}}{\delta t},
$$

where $\frac{d}{d t}=\frac{\partial}{\partial t}+\left(\boldsymbol{u}_{p} \bullet \nabla\right) ; m_{p}, q_{p}$ and $p_{p}$ represent the mass, charge and pressure of charged particles, respectively, $\boldsymbol{E}$ and $\boldsymbol{B}$ are the electric field and magnetic field, respectively; and $\frac{\delta M_{p}}{\delta t}$ is the change in momentum. In an ideal gas, the pressure gradient term is $\nabla p_{p}=\nabla k T_{p} n_{p}$, in which $k$ is the Boltzmann constant and $T_{p}$ is the ion temperature. To solve Eq. (2), we replaced $\frac{d \boldsymbol{u}_{p}}{d t}$ with its zeroorder item $\frac{d \boldsymbol{u}_{p 0}}{d t}$ (Ma and Schunk 1991), in which $\boldsymbol{u}_{p 0}$ is the dominant portion of $\boldsymbol{u}_{p}$. Therefore, the first term on the left side of Eq. (2) can be neglected. Then, the momentum equation becomes:

$$
\frac{k T_{p}}{n_{p} m_{p}} \nabla n_{p}-\frac{q_{p}}{m_{p}}\left(\boldsymbol{E}+\boldsymbol{u}_{p} \times \boldsymbol{B}\right)=\frac{1}{n_{p} m_{p}} \frac{\delta \boldsymbol{M}_{p}}{\delta t} .
$$

The Cartesian coordinate system is established with the $\mathrm{x}$ axis in the eastern direction, $\mathrm{y}$ axis in the northern direction, and $\mathrm{z}$ axis in the vertical direction, and it is assumed that the direction of the magnetic field $\vec{s}=\vec{z} / \sin I \approx \vec{z}$ at high latitudes. Therefore, Eq. (3) can be written as the component form below:

$$
\left\{\begin{array}{c}
\frac{k T_{p}}{m_{p} n_{p}} \frac{\partial n_{p}}{\partial x}-\frac{q_{p} E_{x}}{m_{p}}-\Omega_{p} u_{p y}=\frac{1}{m_{p} n_{p}} \frac{\delta M_{p x}}{\delta t} \\
\frac{k T_{p}}{m_{p} n_{p}} \frac{\partial n_{p}}{\partial y}-\frac{q_{p} E_{y}}{m_{p}}+\Omega_{p} u_{p x}=\frac{1}{m_{p} n_{p}} \frac{\delta M_{p y}}{\delta t} \\
\frac{k T_{p}}{m_{p} n_{p}} \frac{\partial n_{p}}{\partial z}-\frac{q_{p} E_{z}}{m_{p}}=\frac{1}{m_{p} n_{p}} \frac{\delta M_{p z}}{\delta t}
\end{array}\right.
$$

where $\Omega_{p}=q_{p} B / m_{p}$ is the cyclotron frequency of $\mathrm{Ba}^{+}$or $\mathrm{O}^{+}, u_{p x}, u_{p y}$ and $u_{p z}$ are the components of $\boldsymbol{u}_{p}$ in three directions, and $u_{p z}$ is implicit in the last formula of Eq. (4).

The momentum changes of $\mathrm{O}^{+}$or $\mathrm{Ba}^{+}$are as follows:

$$
\begin{aligned}
\frac{\delta M_{\left(O^{+}\right)}}{\delta t}= & m_{\left(O^{+}\right)} n_{\left(O^{+}\right)} \sum_{\alpha} v_{\left(O^{+}\right) \alpha}\left(\boldsymbol{u}_{\alpha}-\boldsymbol{u}_{\left(O^{+}\right)}\right), \\
\frac{\delta M_{\left(B a^{+}\right)}}{\delta t}= & m_{\left(B a^{+}\right)} n_{\left(B a^{+}\right)} \sum_{\alpha} v_{\left(B a^{+}\right) \alpha}\left(\boldsymbol{u}_{\alpha}-\boldsymbol{u}_{\left(B a^{+}\right)}\right) \\
& +m_{\left(B a^{+}\right)} n_{s} \sigma\left(\boldsymbol{u}_{s}-\boldsymbol{u}_{\left(B a^{+}\right)}\right),
\end{aligned}
$$

where $v_{O^{+} \alpha}$ and $v_{B a^{+} \alpha}$ are the collision frequencies between $\mathrm{O}^{+} / \mathrm{Ba}^{+}$and other particles $\alpha$ ( $\alpha$ represents charged particles and neutral particles), and $\boldsymbol{u}_{s}$ and $n_{s}$ are the velocity and number density of the neutral atoms, respectively. In addition to the change in momentum due to collision, momentum change due to the source term, $m_{\left(B a^{+}\right)} n_{s} \sigma\left(\boldsymbol{u}_{s}-\boldsymbol{u}_{\left(B a^{+}\right)}\right)$, should also be included for barium ions. Due to the binding effect of the geomagnetic field, the difference between charged particles in velocity perpendicular to $B$ is usually small, so the collision terms associated with it can be ignored (Schunk and Szuszczewicz 1988), and the electron inertia is also ignored.

The electric field is calculated by $\boldsymbol{E}=\boldsymbol{E}_{0}-\nabla \varphi$, where $\boldsymbol{E}_{0}$ is the constant electric field of the background, $\varphi$ is the disturbance of the electrostatic potential, and $\nabla \varphi$ can be approximately calculated by the following formula (Ma and Schunk 1993):

$$
\nabla \varphi=\left(k T_{e} / e\right) \nabla \ln \left(n_{e}\right),
$$

where $T_{e}$ is the electron temperature, $e$ is the Napierian base, and the electron density $n_{e}$ is calculated by the quasi-neutral condition, $n_{e}=n_{\left(B a^{+}\right)}+n_{\left(O^{+}\right)}$.

By substituting Eqs. $(5,6,7)$ into Eq. $(4)$, the drift velocities of charged particles in the three directions can be obtained.

\section{Diffusion of neutral particles}

After being released in the ionosphere, the photoionization and recombination reaction of neutral barium as well as the oxidation reactions will occur. The main reaction process is described as follows:

$$
\begin{aligned}
& \mathrm{Ba}+h v \stackrel{\sigma}{\rightarrow} \mathrm{Ba}^{+}+e^{-} \\
& 2 \mathrm{Ba}+\mathrm{O}_{2} \stackrel{k_{c}}{\rightarrow} 2 \mathrm{BaO} \\
& k_{c}=0.9 \times 10^{-10} \mathrm{~cm}^{3} \mathrm{~s}^{-1},
\end{aligned}
$$

where $k_{c}$ is the reaction coefficient.

Taking the photoionization and oxidation losses into account, the general equation satisfied by the neutral particles can be described as follows:

$$
\frac{\partial n_{s}}{\partial t}+\nabla \cdot\left(\boldsymbol{u}_{s} n_{s}\right)=\nabla \cdot\left(D \nabla n_{s}\right)-L_{s}
$$

where $L_{s}=\sigma n_{s}+k_{c} n_{\mathrm{O}_{2}} n_{s}$ is the loss term, in which $\sigma n_{s}$ represents photoionization loss and $k_{c} n_{\mathrm{O}_{2}} n_{s}$ represents oxidation loss. $D$ is the diffusion coefficient, which can be calculated by the following formula (Bleecker et al. 2004):

$$
D=\frac{3}{16} \frac{\left(2 \pi k T / \mu_{s} O\right)^{1 / 2}}{n_{O} \pi\left(r_{s}+r_{O}\right)^{2}},
$$

where $n_{O}$ is the number density of atomic oxygen, and $T$ is the neutral temperature. $\mu_{s O}=\frac{m_{O} m_{s}}{m_{s}+m_{O}}$ is the reduced mass, and $m_{s}, m_{O}, r_{s}$ and $r_{O}$ are the mass and atomic radius of released neutral and oxygen atoms, respectively. 
The approximate solution of Eq. (8) has been derived (Hu et al. 2012): flow of the numerical algorithm is shown in Fig. 1, and the simulation process can be summarized as follows:

$$
n_{s}(r, t)=\frac{N_{0}}{\pi^{3 / 2}\left(4 D t+r_{0}^{2}\right)\left(4 D t+\varepsilon^{2} r_{0}^{2}\right)^{1 / 2}} \times \exp \left(-\frac{\left(x-x_{0}-\int u_{x} d t\right)^{2}+\left(y-y_{0}-\int u_{y} d t\right)^{2}}{4 D t+r_{0}^{2}}-\frac{\left(z-z_{0}-\int u_{z} d t\right)^{2}}{4 D t+\varepsilon^{2} r_{0}^{2}}-\sigma t-k_{c} n_{O_{2}} t\right),
$$

where $\left(x_{0}, y_{0}, z_{0}\right)$ is the initial release center of the cloud, $u_{x}, u_{y}$ and $u_{z}$ are the velocity components of $\boldsymbol{u}_{s}$ in three directions, $r_{0}$ is the initial characteristic radius, $N_{0}$ is the total number of released particles, and $\varepsilon$ represents the shape factor.

In the ionospheric $\mathrm{F}$ region, the neutral barium cloud decelerates under the impact of collisions with the background particles, and its velocity changes with time can be approximately expressed as:

$$
\boldsymbol{u}_{s}(t)=\left(\boldsymbol{v}_{0}-\boldsymbol{u}_{n}\right) e^{-v_{s} t},
$$

where $\boldsymbol{v}_{0}$ is the initial release velocity of the neutral cloud, $\boldsymbol{u}_{n}$ is the background neutral wind, and $v_{s}$ is the damping coefficient of the decelerating motion of the cloud due to collisions, which can be obtained according to elastic collision theory (Zhang et al. 2019):

$$
v_{s}=n_{O}\left(r_{s}+r_{O}\right)^{2} \pi \sqrt{\frac{8 k T}{\pi \mu_{s O}}} \frac{m_{O}}{m_{s}+m_{O}} .
$$

By time integrating Eq. (11), the change in the position of the cloud center can be obtained:

$$
r_{c}(t)=\boldsymbol{u}_{n} t+\int_{0}^{t}\left(\boldsymbol{\nu}_{0}-\boldsymbol{u}_{n}\right) e^{-v_{s} t} d t
$$

The spatial distribution of particles in the released region can then be obtained by solving Eqs. (1, 2, 3, 4, 5, $6,7,8,9,10,11,12,13)$ numerically.

\section{Results and discussion}

In this section, the evolution characteristics of artificial plasma clouds under various release conditions were investigated, including different release altitudes $(220 \mathrm{~km}$ and $300 \mathrm{~km})$, different release masses $(1 \mathrm{~kg}, 10 \mathrm{~kg}$ and $100 \mathrm{~kg})$, different initial ionization rates $(0 \%, 20 \%$ and $80 \%$ ), and different release velocities. The ionospheric disturbances of barium and cesium were also compared.

The ambient atmospheric density, ionospheric particle density, temperature, magnetic field intensity and other initial conditions can be obtained by the atmospheric model MSIS-E-90, International Reference Ionosphere model (IRI-2016), and International Geomagnetic Reference Field model (IGRF-13). An equivalent extrapolation boundary condition is used at all boundaries. The design
1. The release parameters and background ionospheric parameters are set;

2. The distribution of neutrals is calculated according to the neutral diffusing model ("Diffusion of neutral particles" section);

3. The drift velocity is calculated according to the momentum equation (Eq. (2) in "Diffusion of charged particles" section);

4. The particle number density distribution can be obtained by solving the continuity equation (Eq. (1) in "Diffusion of charged particles" section);

5. Steps $2-4$ are repeated until the time limit is reached.

\section{Effects of release altitude}

The ambient temperature, magnetic field, and number density of neutral particles and charged particles in the ionosphere vary with altitude, so the collision frequency and diffusion coefficient vary with altitude. At low altitudes, the diffusion coefficient of barium is small due to the high concentration of atomic oxygen, molecular oxygen and other particles, and the chemical consumption of barium is high, resulting in a relatively small plasma

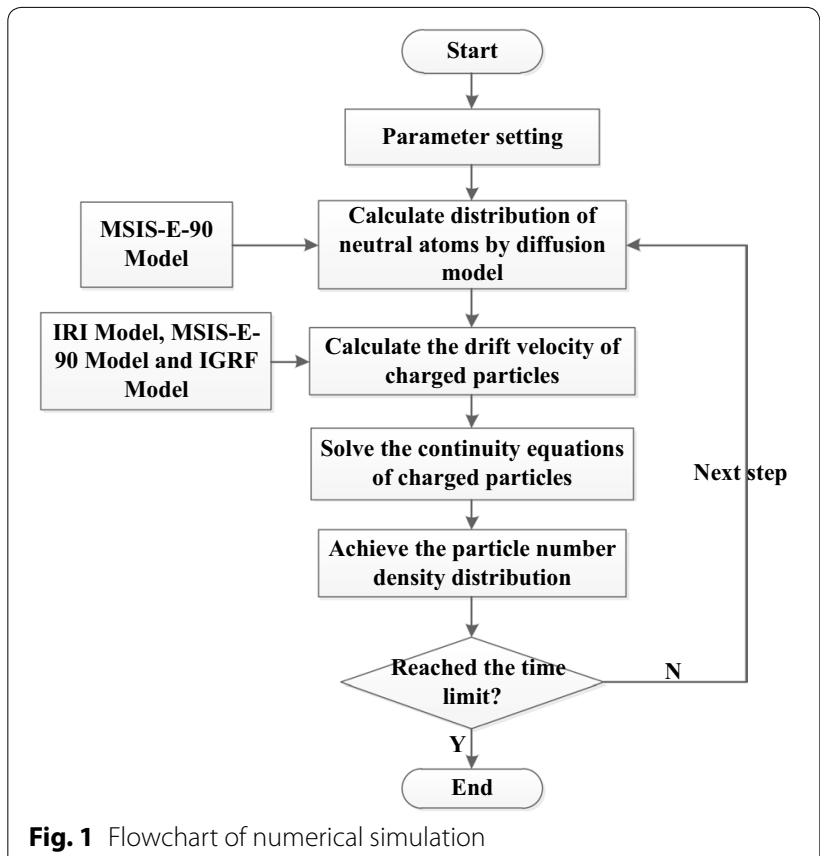


cloud. However, if the chemical is released at a very high altitude, rapid diffusion leads to a sharp reduction in the concentration of the released substance, and the background ionospheric particles cannot be significantly affected, which is not conducive to observation. For this reason, release altitudes of $220 \mathrm{~km}$ and $300 \mathrm{~km}$ are selected in our simulation, where the diffusion velocity is moderate and the disturbing effect on the background ionosphere is obvious, which is more convenient for experimental observation.

The distribution of the density and temperature of the ambient particles, and the diffusion coefficient and damping coefficient of barium atoms, and the meridional and zonal winds obtained in Horizontal Wind Model 07 are shown in Fig. 2.

Figures 3a, 4a show the release results at $220 \mathrm{~km}$ and $300 \mathrm{~km}$, respectively. The profile of barium ions (Figs. 3a, $4 \mathrm{a})$, background oxygen ions (Figs. 3b, 4b) and electron number density (Figs. 3c, 4c) are shown in the subgraphs. Due to the binding effect of the geomagnetic field, the expansion of the plasma cloud across $\boldsymbol{B}$ is constrained. In the direction along the magnetic field, the movement of the barium ion cloud is not restricted, so the plasma cloud will be gradually tied to the magnetic field, and stretched into an elliptical structure along the direction of the magnetic field. The momentum of $\mathrm{Ba}^{+}$and $\mathrm{O}^{+}$are coupled together because of collision, and barium ions transfer the kinetic energy to oxygen ions, which pushes the oxygen ions to move along $\boldsymbol{B}$, forming an oxygen ion density hole in the release center. On the other hand, the outflowing $\mathrm{O}^{+}$slows down under the effects of the background thermal and pressure gradients, which creates two density bumps on both sides of the $\mathrm{Ba}^{+}$cloud along $\boldsymbol{B}$. This phenomenon is called the snowplow effect (Ma and Schunk 1991), which has been detected by a high-resolution incoherent scatter radar in the Spacelab 2 upper atmospheric modification experiment (Bernhardt et al. 1988).

Figures $3 \mathrm{~d}$, e, $4 \mathrm{~d}$, e show the number density and velocity of electrons as a function of altitude and time, respectively. Due to the high collision frequency at low altitudes, the expansion of neutral barium clouds is greatly restrained, and the plasma clouds expand more slowly, so the plasma clouds are mainly concentrated in a relatively small area. Furthermore, Figs. 3d, 4d show that the peak number density of electrons at the release center decreases more slowly at low altitudes. At a high altitude, with a lower collision frequency and higher diffusion coefficient, the expansion of neutral clouds is faster, and the plasma clouds stretch faster along the magnetic field, so the plasma clouds have a larger radius than those at low altitudes. As can be seen from Figs. 3e, 4e, when barium is released at $220 \mathrm{~km}$ and $300 \mathrm{~km}$, the maximum diffusion velocities of electrons that can be achieved at $t=30 \mathrm{~s}$ are $466 \mathrm{~m} / \mathrm{s}$ and $965 \mathrm{~m} / \mathrm{s}$, respectively.

When considering a nonuniform ionosphere, the diffusion coefficient and damping coefficient change with height, and the background density gradient also affects the ion motion. The motion of artificial ion clouds along the magnetic field and its snowplow effect on ambient oxygen ions are similar to previous simulation results in which the background ionosphere is assumed to be

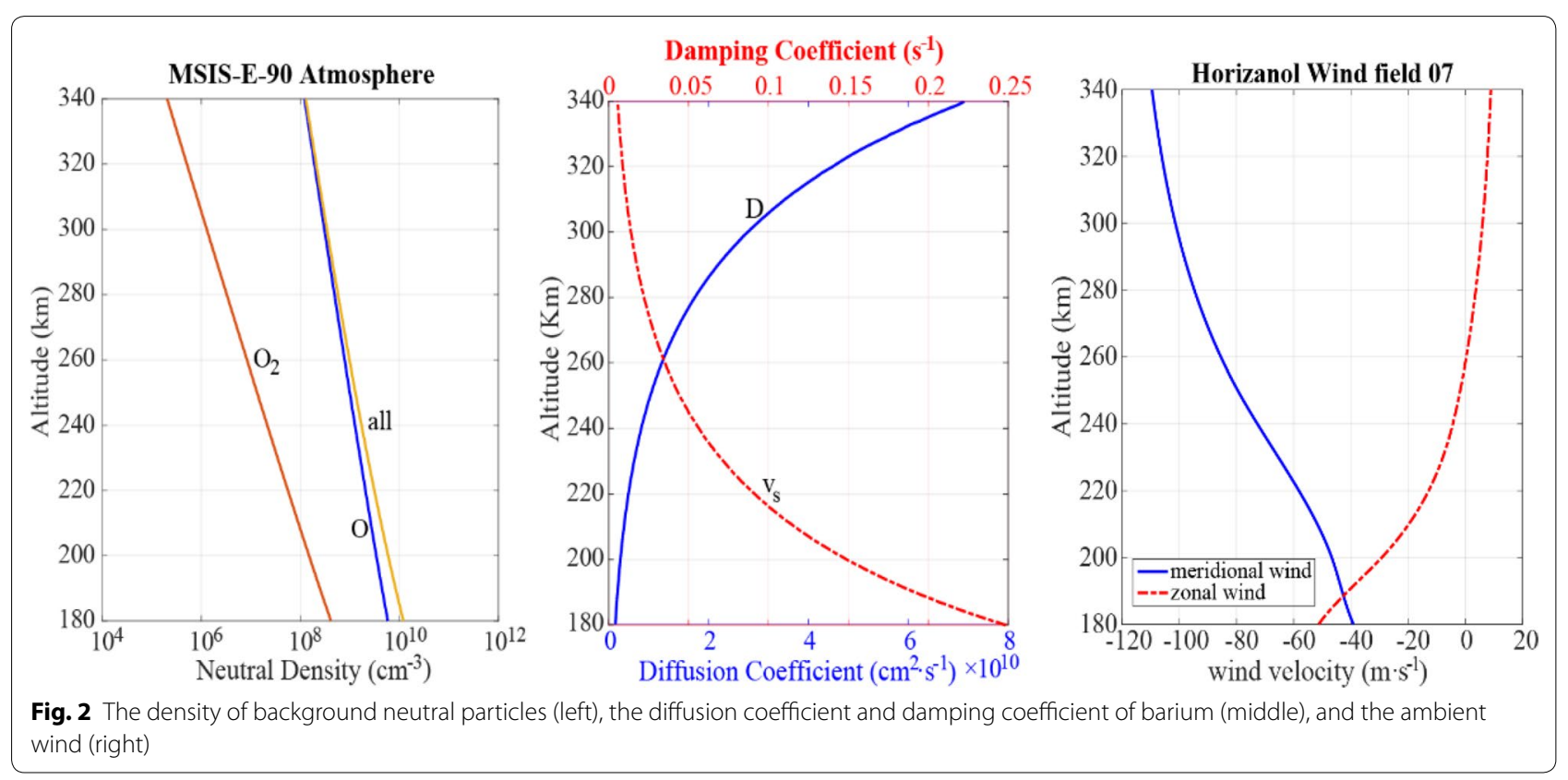




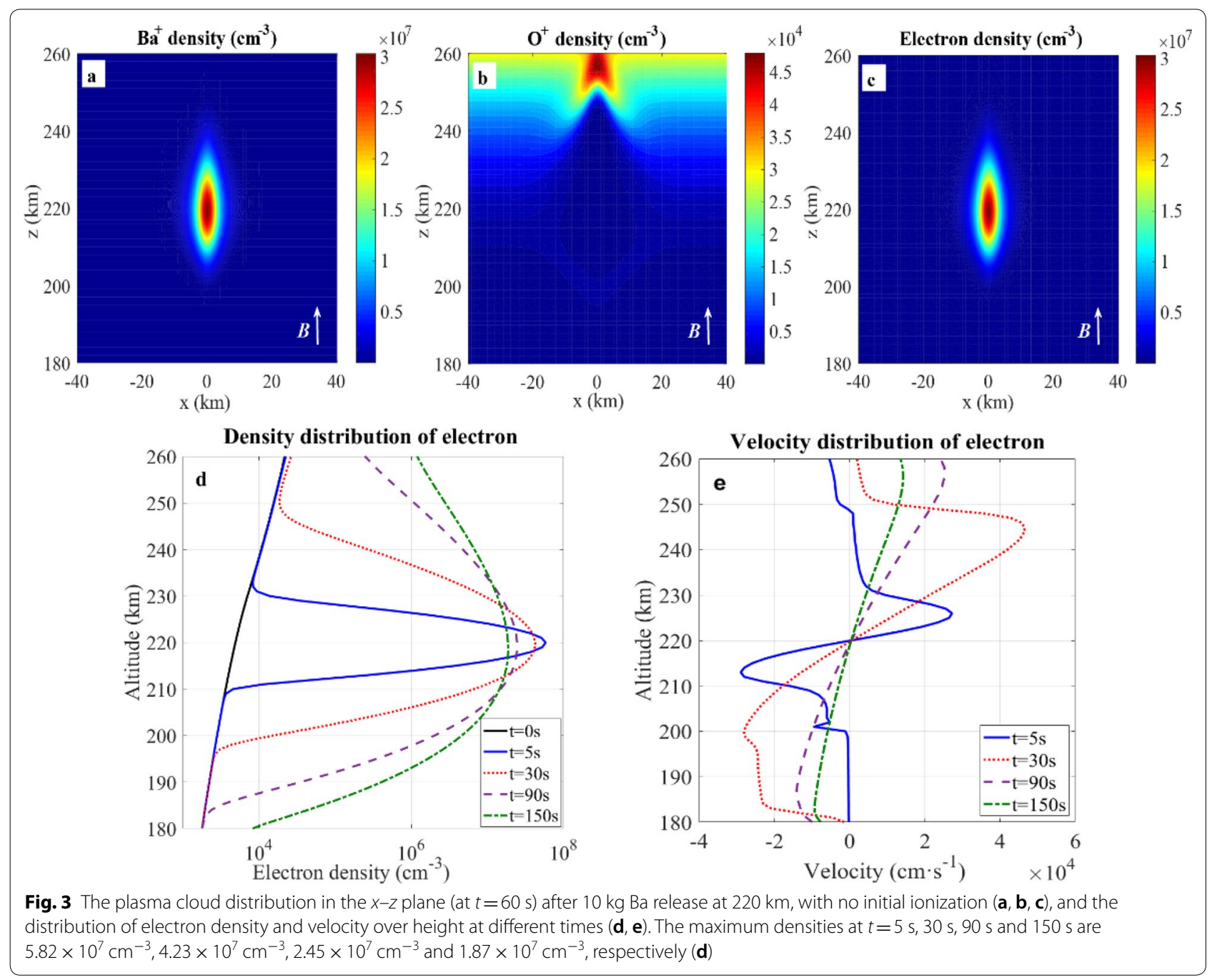

uniform (Gatsonis and Hastings 1991; Ma and Schunk 1991, 1993). The difference is that the asymmetric structure of the artificial ion cloud appears in the vertical direction, which can be ascribed to the asymmetry of the collision frequency and diffusion coefficient, leading to a plasma cloud with a longer top and shorter bottom. In addition, the snowplow effect of $\mathrm{O}^{+}$is also asymmetric on both sides of the expansion cloud due to the influence of the background density gradient. Based on the results without considering the ambient wind field (not shown), the effect of the ambient wind field is not significant because the speed of the ambient neutral wind is approximately tens of meters per second, which is much smaller than the expansion velocity of the plasma cloud (on the order of kilometers per second). Qualitatively, the morphology and evolution characteristics of the artificial plasma cloud are in agreement with the observations in previous space experiments (Haerendel et al. 1967; Foppl et al. 1967; Bernhardt et al. 1987; Huba et al. 1992).

\section{Effects of initial cloud density}

In this section, we analyze the effects of different initial cloud densities on the evolution of artificial plasma clouds. Changes in release masses and initial ionization rates can both change the initial densities of the cloud.

For a fixed initial radius, different release masses mean different initial cloud densities. Qualitatively speaking, the results of ionospheric disturbances with different release amounts are similar. It can be seen from Fig. 5 that with a larger release mass, the number density of the plasma clouds will be higher, and the enhanced pressure gradient enhances the expansion kinetic energy of neutral clouds, leading to a stronger disturbance of background oxygen ions and electrons. Additionally, a large release mass causes a larger ionospheric disturbance area and a longer duration of disturbance.

In an actual release experiment, it often takes some time for the cloud to reach the initial state of our simulation (which is generally called the average collision time), 


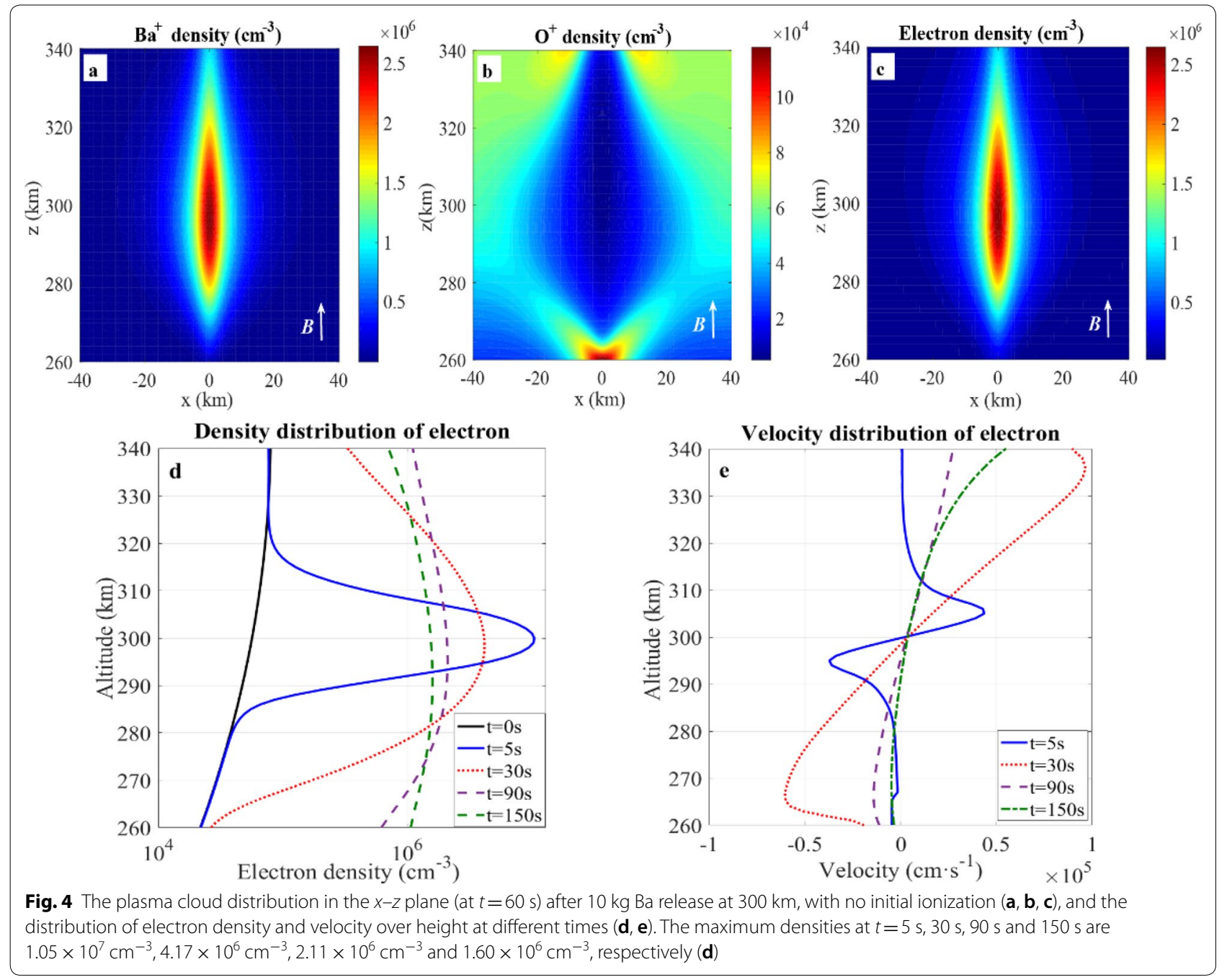

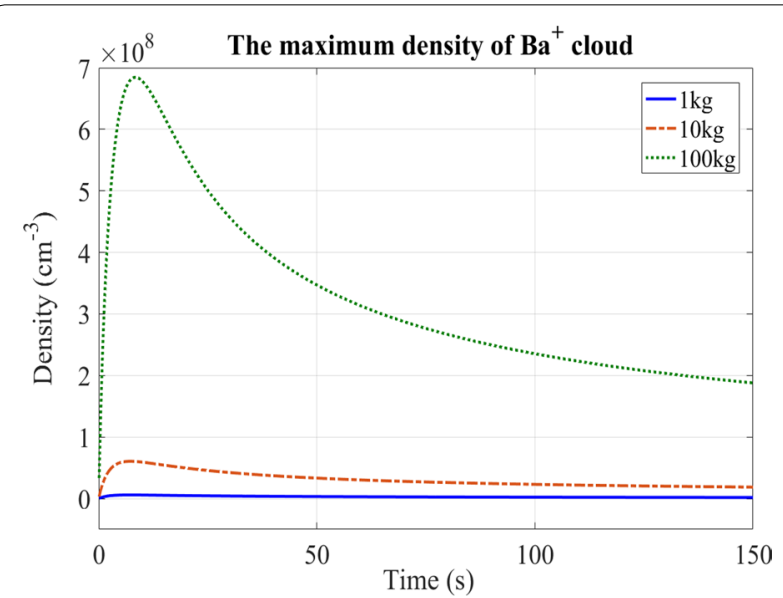

Fig. 5 The density of artificial plasma clouds at the release center varies with time under different release masses. The maximum densities of $\mathrm{Ba}^{+}$clouds are $5.93 \times 10^{6} \mathrm{~cm}^{-3}, 6.06 \times 10^{6} \mathrm{~cm}^{-3}$ and $6.84 \times 10^{6} \mathrm{~cm}^{-3}$ when $1 \mathrm{~kg}, 10 \mathrm{~kg}$, and $100 \mathrm{~kg}$ of barium are released, respectively so that a few barium atoms may have been ionized before the beginning of the simulation, leading to a difference between the initial number of barium atoms and the total number of barium atoms released, which is called the initial ionization rate. In addition, due to the different release techniques (thermal release, explosion release, etc.) used in the active release experiment, the ionization rates of neutral clouds at the beginning of release also vary greatly.

Figure 6 shows the simulation results with initial ionization rates of $0 \%, 20 \%$ and $80 \%$. The plasma cloud consists of two parts: one part is the high-density part due to initial ionization, and this part will become longer and narrower over time, because it has little movement across the magnetic field except for the initial inertial motion (e.g., the thermal expansion after release from the canister and the velocity generated by the suborbital motion of the sounding rocket), which is soon be captured by magnetic field. Another part of the plasma cloud comes from 


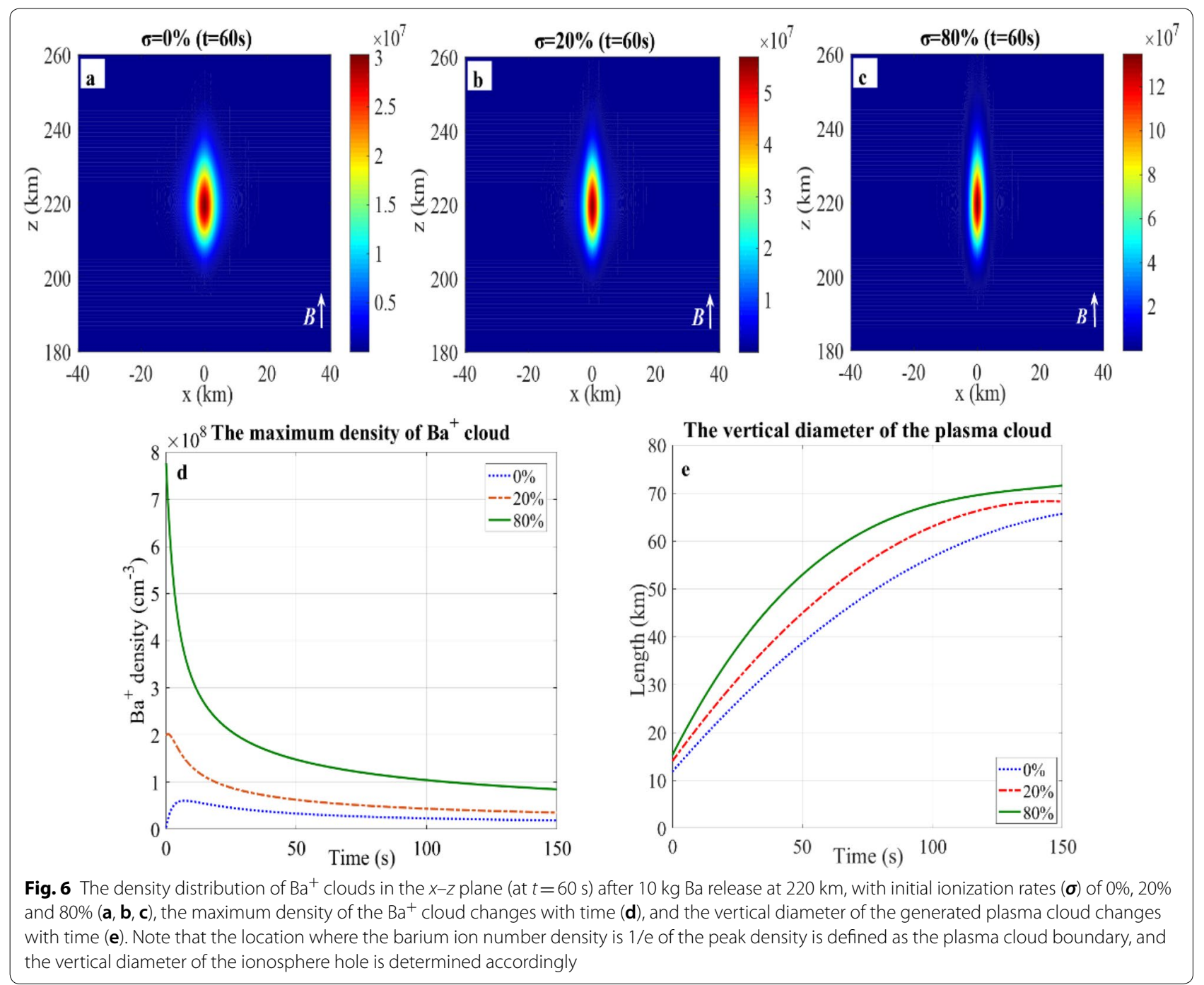

the continual photoionization of expanding barium neutrals. At the initial stage after release, a steeper density gradient results in a faster expansion of the plasma cloud along $\boldsymbol{B}$. As seen from Fig. 6, with the increase in the initial ionization rate, the distribution of barium ion clouds become increasingly concentrated, and the numerical dissipation brought by the numerical simulation process decreases, so the highest density of $\mathrm{Ba}^{+}$increases, but the plasma density decreases faster. Figure $6 \mathrm{~d}$, e shows that the maximum $\mathrm{Ba}^{+}$cloud densities are $7.77 \times 10^{8} \mathrm{~cm}^{-3}$, $2.02 \times 10^{8} \mathrm{~cm}^{-3}$ and $6.06 \times 10^{7} \mathrm{~cm}^{-3}$ with initial ionization rates of $0 \%, 20 \%$ and $80 \%$, respectively, and the diameters of the plasma clouds with initial ionization rates of $0 \%, 20 \%$ and $80 \%$ are $43 \mathrm{~km}, 50 \mathrm{~km}$ and $57 \mathrm{~km}$ at $t=60 \mathrm{~s}$, respectively. At the same time, the steeper density gradient makes the barium cloud stretch faster along the magnetic field, so the vertical diameter of the plasma cloud also increases rapidly, and the sheet-like structure of the plasma cloud along the magnetic field is more obvious. As time passes, the density difference of $\mathrm{Ba}^{+}$at the release center caused by different initial ionization rates decreases.

\section{Effects of release velocity}

We also considered the evolution characteristics of released clouds with different initial release velocities. The cloud evolution results with initial velocity perpendicular to $\boldsymbol{B}$ and initial velocity parallel to $\boldsymbol{B}$ were simulated, with a velocity of $2 \mathrm{~km} / \mathrm{s}$. It should be noted that, for the release with initial velocity perpendicular to magnetic field, we take the release point located at $[-20$, $0,0] \mathrm{km}$; for the release with initial velocity parallel to magnetic field, we take the release point located at $[0,0$, $-20] \mathrm{km}$.

Figure 7 shows the evolution result of a cloud released with an initial velocity perpendicular to 

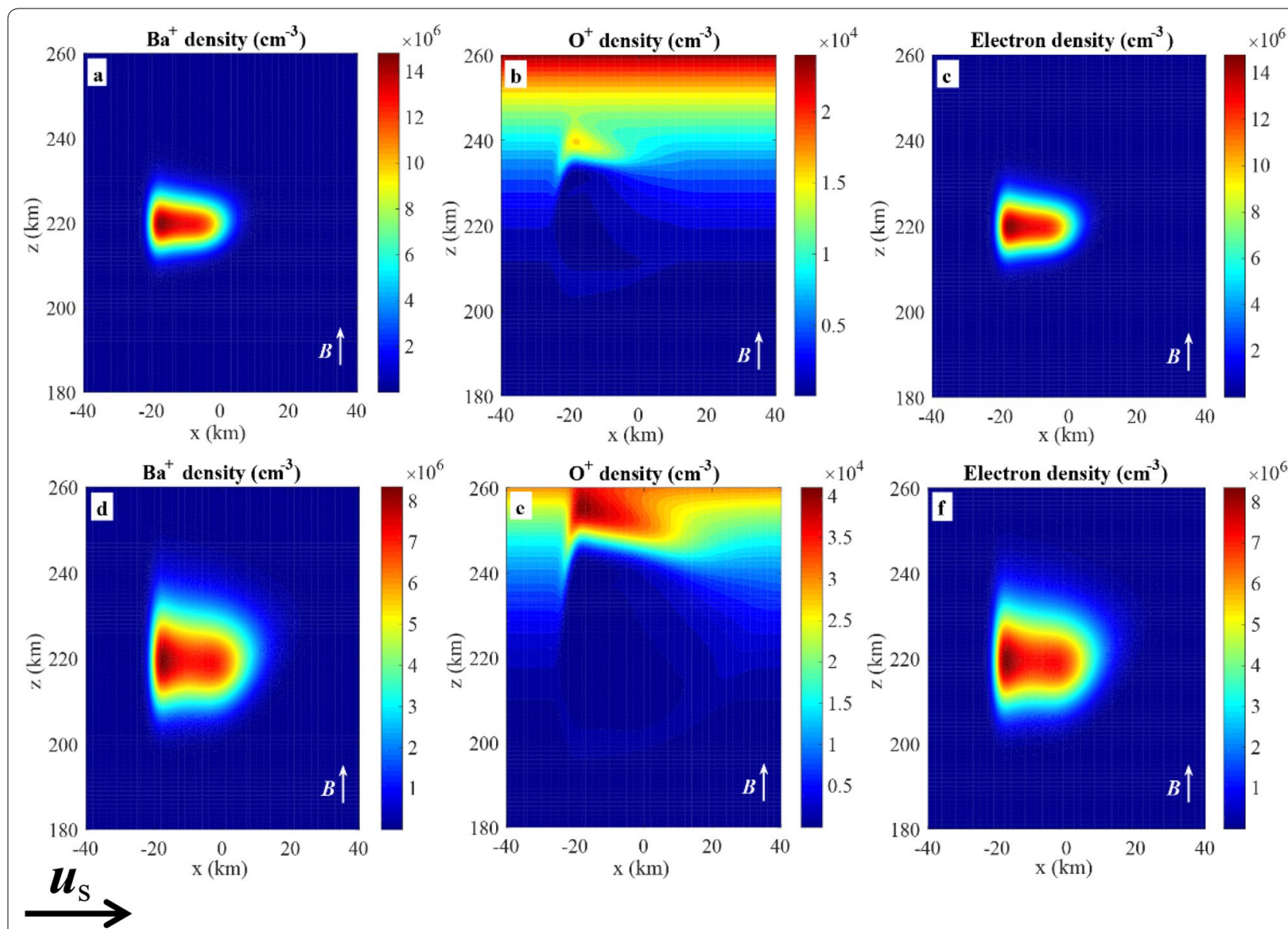

Fig. 7 The plasma cloud distribution in the $x-z$ plane at $t=20 \mathrm{~s}(\mathbf{a}, \mathbf{b}, \mathbf{c})$ and $60 \mathrm{~s}(\mathbf{d}, \mathbf{e}, \mathbf{f})$ after $10 \mathrm{~kg}$ Ba release at $220 \mathrm{~km}$, and the injection velocity $\boldsymbol{u}_{\mathrm{s}}$ is $2 \mathrm{~km} / \mathrm{s}$ perpendicular to $\boldsymbol{B}$

$\boldsymbol{B}$. The expansion of the plasma cloud along $\boldsymbol{B}$ and its snowplow effect on $\mathrm{O}^{+}$are very similar to those of a stationary release. However, since the volume of the $\mathrm{Ba}^{+}$cloud is larger than that in the former case, the number density of the $\mathrm{Ba}^{+}$cloud is lower. Due to the movement of neutral barium clouds, an ionic tail will be generated behind them from photoionization in the early stage, but the $\mathrm{Ba}^{+}$cloud still becomes a sheet-like structure eventually because the motion perpendicular to $\boldsymbol{B}$ is constrained. The $\mathrm{Ba}^{+}$cloud decelerated rapidly in response to the magnetic field, while the neutral barium cloud was not affected by the magnetic field; thus, the barium ion cloud was slowly separated from barium neutral clouds (not shown). In addition, there are still two $\mathrm{O}^{+}$density enhancement regions and one $\mathrm{O}^{+}$density depletion region in the background ionosphere, and it can be seen that a small number of oxygen ions are pushed to the front of the cloud due to momentum transfer, forming $\mathrm{O}^{+}$density enhancement regions at both sides in front of the cloud.
As shown in Fig. 8, when the neutral barium cloud is released with a velocity along $\boldsymbol{B}$, at the early stage, the snowplow effect of the ion cloud creates an $\mathrm{O}^{+}$density hole on the back side of the expanding $\mathrm{Ba}^{+}$cloud and an $\mathrm{O}^{+}$density bump at the front. Compared with the cases without injection velocity and with a release velocity perpendicular to $\boldsymbol{B}$, this case features a much greater $\mathrm{O}^{+}$ density enhancement in front of the plasma cloud, and no density enhancement appears behind the plasma cloud. While the initial velocity of the barium neutrals is parallel to the background magnetic field and although the movement of the barium ion cloud is not affected by the $\boldsymbol{j} \times \boldsymbol{B}$ force, with the high concentration of background particles, the motion of the ion cloud still slows down due to collisions. At $t=20 \mathrm{~s}$, the plasma cloud has moved approximately $12 \mathrm{~km}$. Moreover, because of the existence of Coulomb collision between charged particles, the damping coefficient of the ion cloud is higher than that of the neutral cloud, which eventually leads to the separation of the ion cloud and neutral cloud. 


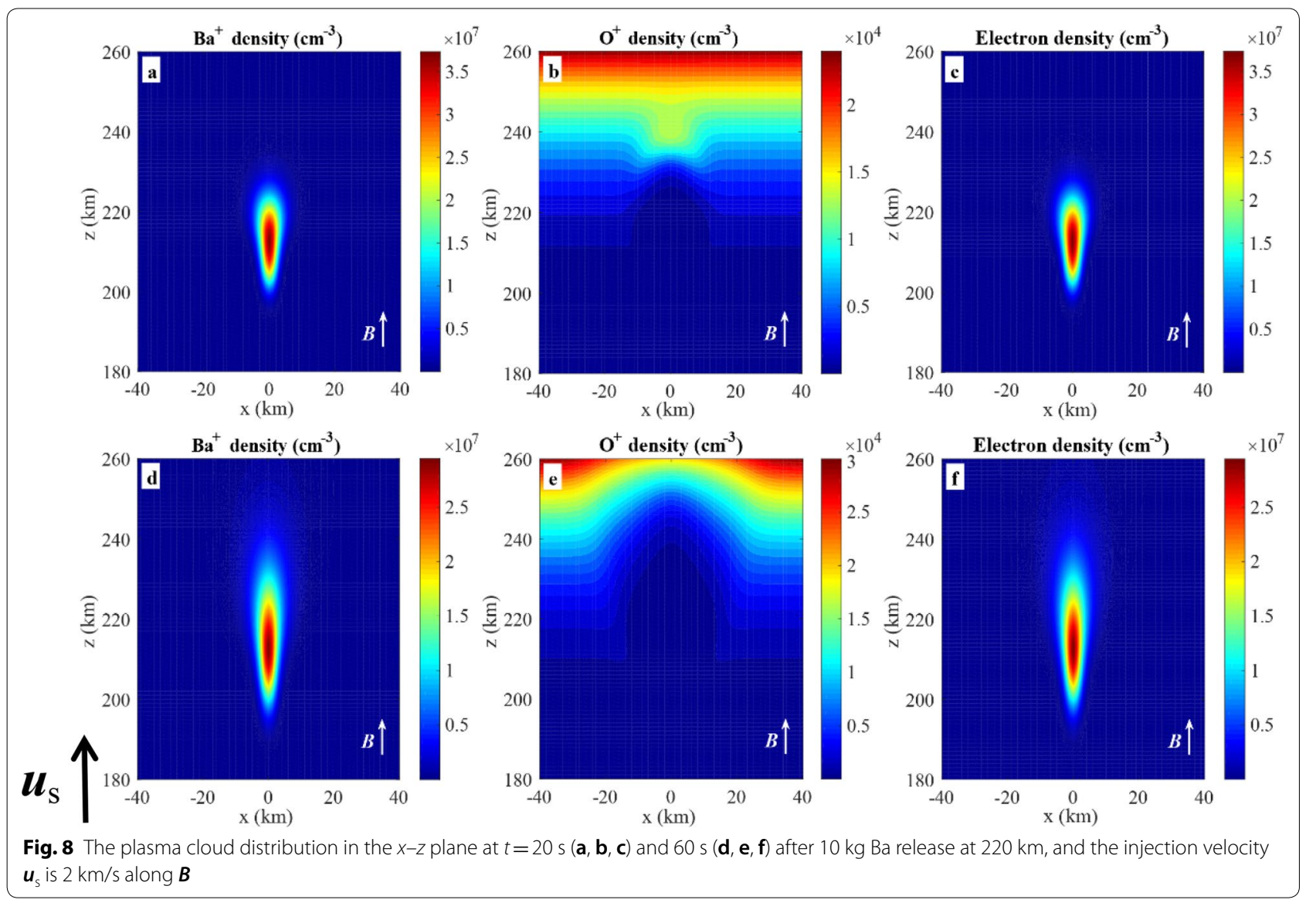

\section{Cesium release}

Although the thermal ionization of cesium is prone to occur due to its low ionization potential, thermal ionization is insignificant compared to photoionization under conditions of strong sunlight, so the thermal ionization process of cesium is not discussed here. The comparison of the diffusion coefficient and damping coefficient of barium and cesium is shown in Fig. 9. The diffusion coefficient of $\mathrm{Ba}$ is slightly larger than that of $\mathrm{Cs}$, but its damping coefficient is smaller than that of Cs.

Figure 10 shows the simulation results of $\mathrm{Cs}$ with the same number of molecules released. Except for the type of material released, other parameters are the same as in Fig. 3. Qualitatively speaking, the expansion characteristics of $\mathrm{Cs}^{+}$and $\mathrm{Ba}^{+}$and the disturbance effect on background $\mathrm{O}^{+}$are similar. Since the diffusion coefficient of cesium is smaller, the barium cloud expands more rapidly and covers a wider area than the cesium cloud, but the ionization yield of cesium is higher than that of barium under the same release mass due to the higher photoionization rate of cesium. The cesium ion cloud is denser than the barium ion cloud, so the cesium ion cloud has a larger expansion speed, as seen from
Figs. 3e, 10e. The maximum vertical velocities of electrons at $t=5 \mathrm{~s}$ are $289 \mathrm{~m} / \mathrm{s}$ and $613 \mathrm{~m} / \mathrm{s}$, respectively. At $t=60 \mathrm{~s}$, the peak number density of cesium ion clouds reaches $4.48 \times 10^{7} \mathrm{~cm}^{-3}$, which is nearly one and a half times that of barium ion clouds $\left(3.03 \times 10^{7} \mathrm{~cm}^{-3}\right)$ under the same conditions. In addition, the collision frequency of $\mathrm{Cs}^{+}-\mathrm{O}^{+}$is greater than that of $\mathrm{Ba}^{+}-\mathrm{O}^{+}$, and the snowplow effect of $\mathrm{Cs}^{+}$is stronger than that of $\mathrm{Ba}^{+}$, resulting in larger oxygen ion density holes and bumps.

\section{Conclusions}

In this study, the evolution characteristics of artificial plasma clouds under different release conditions were systematically studied based on a three-dimensional twospecies model. The influences of different release altitudes, masses, initial ionization rates, release velocities and release substances on the evolution of the artificial plasma cloud were discussed. Based on the simulation results, the conclusions can be summarized below:

1. When a nonuniform ionosphere with altitude is assumed, the motion of artificial ion clouds along the magnetic field and their snowplow effect on 

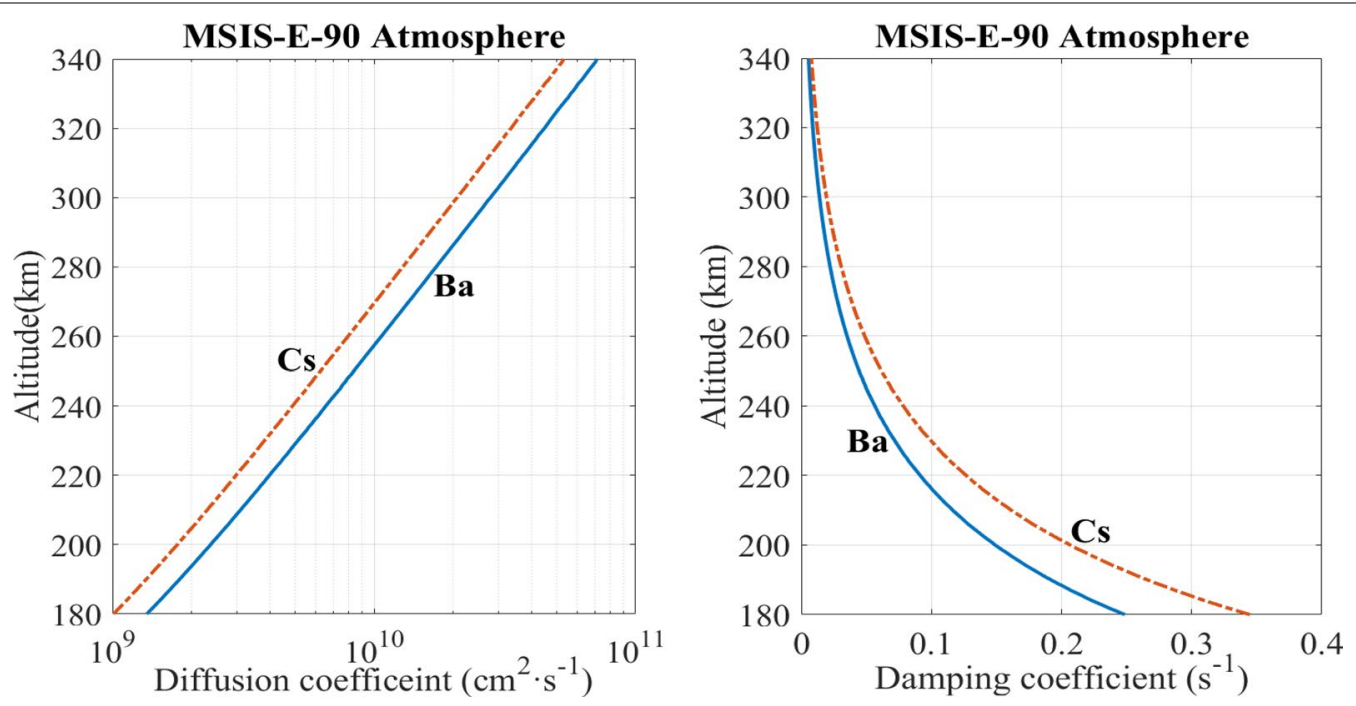

Fig. 9 Comparison of the diffusion coefficient (left) and damping coefficient (right) of Cs and Ba
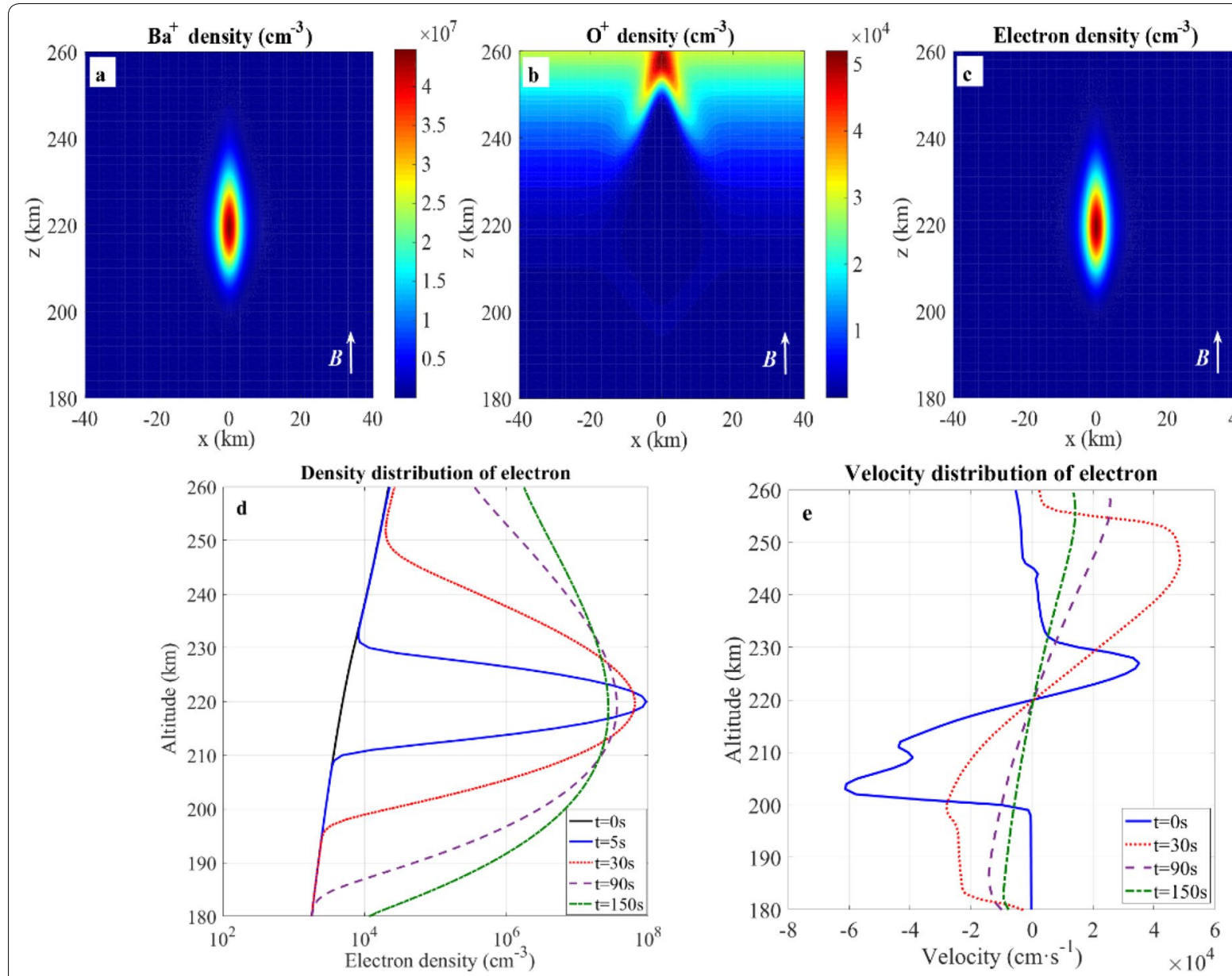

Fig. 10 The plasma cloud distribution in the $x-z$ plane (at $t=60 \mathrm{~s})$ after $10 \mathrm{~kg}$ Cs release at $220 \mathrm{~km}$, with no initial ionization $(\mathbf{a}, \mathbf{b}, \mathbf{c})$, and the distribution of electron density and velocity over height at different times (d, e). The maximum densities at $t=5 \mathrm{~s}, 30 \mathrm{~s}, 90 \mathrm{~s}$ and $150 \mathrm{~s}$ are $9.27 \times 10^{7} \mathrm{~cm}^{-3}, 6.44 \times 10^{7} \mathrm{~cm}^{-3}, 3.59 \times 10^{7} \mathrm{~cm}^{-3}$ and $2.72 \times 10^{7} \mathrm{~cm}^{-3}$, respectively (d) 
background oxygen ions are similar to the results obtained when considering a uniform ionosphere. The difference is that an asymmetric structure of the artificial ion cloud appears in the vertical direction, which can be ascribed to the asymmetry in the collision frequency. In addition, the snowplow effect of oxygen ions is asymmetric on both sides of the expansion cloud due to the influence of the background density gradient.

2. At high altitudes, due to the low collision frequency between particles, the neutral clouds expand rapidly, and the artificial ion cloud has a large volume and is stretched rapidly along the magnetic field. The disturbance to the background oxygen ions is strong, but the disturbance recovers rapidly.

3. Essentially, different release masses and initial ionization rates can both change the initial density of the plasma cloud, and have a similar consequence on cloud evolution in the early stage. The difference is that the disturbance in the ionosphere will last longer with a larger release mass, while with higher initial ionization rates, the density gradient of the plasma cloud will be steeper, so that the plasma stretches faster along $\boldsymbol{B}$, leading to a plasma cloud with a more obviously elongated structure.

4. The initial release velocity can change the motion and overall distribution of the cloud. For a cloud released with an initial velocity perpendicular to $\boldsymbol{B}$, the cloud becomes stretched in the same direction as the initial velocity without moving, and a small amount of oxygen ions is pushed away in front of the cloud due to momentum transfer. When the initial release velocity is parallel to $B$, the $\mathrm{O}^{+}$density enhancement in front of the cloud is much greater than that behind the cloud, and the plasma cloud moves in same direction. Barium releases with initial velocities both perpendicular and parallel to $B$ have a strong disturbance on background particles, eventually resulting in the separation of the ion cloud and the neutral cloud.

5. Qualitatively, the evolution characteristics of $\mathrm{Cs}^{+}$and $\mathrm{Ba}^{+}$and their effects on background $\mathrm{O}^{+}$are similar. Due to the large diffusion coefficient of barium, the expansion of barium clouds is more rapid, and the coverage area of $\mathrm{Ba}^{+}$clouds is wider, but the density of $\mathrm{Cs}^{+}$clouds is higher than that of $\mathrm{Ba}^{+}$clouds when the same mass is released because of the large photoionization rate of cesium. Moreover, the snowplow effect of $\mathrm{Cs}^{+}$is stronger than that of $\mathrm{Ba}^{+}$, and the oxygen ion density disturbance caused by cesium is stronger.

It is worth noting that the formation of striations, as observed in many experiments, does not appear in our simulation (Zalesak et al. 1988; Goldman et al. 1976; Kelley and Livingston 2003). The main reason is that we assumed that the density distribution of the neutral cloud is Gaussian and the overall density distribution is smooth, so that the $\boldsymbol{E} \times \boldsymbol{B}$ gradient instability did not occur, which is generally thought to be the main cause of striations. In addition, the lack of a varying background $\boldsymbol{E}$ field may be another reason for the absence of striations.

\section{Abbreviations \\ IRI: International Reference lonosphere; IGRF: International Geomagnetic Reference Field.}

\section{Acknowledgements}

We are thankful to the reviewers that helped us to improve the quality of the paper, and a special thanks to all the persons that helped us to complete this work.

\section{Authors' contributions}

XZ worked on theory development, simulations and manuscript writing. YH and $B N$ helped with the analyses and in preparing the manuscript. $Z Z$ and $Y Z$ worked on theory development, discussion and supervision of the study. All authors read and approved the final manuscript.

\section{Funding}

This research received no external funding.

Availability of data and materials

Simulation data can be provided upon request.

\section{Competing interests}

The authors declare that they have no competing interests.

Received: 12 March 2020 Accepted: 19 November 2020

Published online: 30 November 2020

\section{References}

Bernhardt PA, Roussel-Dupre RA, Pongratz MB, Haerendel G, Valenzuela A, Gurnett DA et al (1987) Observations and theory of the AMPTE magnetotail barium releases. J Geophys Res 92(A6):5777. https://doi.org/10.1029/ ja092ia06p05777

Bernhardt PA, Swartz WE, Kelly MC, Sulzer MP, Noble ST (1988) Spacelab 2 upper atmospheric modification experiment over Arecibo, 2, plasma dynamics. Astrophys Lett Comm 27(3):183

Bleecker KD, Bogaerts A, Gijbels R, Goedheer W (2004) Numerical investigation of particle formation mechanisms in silane discharges. Phys Rev $E$ 69(5):056409. https://doi.org/10.1103/PhysRevE.69.056409

Caton RG, Pedersen TR, Groves KM, Hines J, Cannon PS, Jackson-Booth N et al (2017) Artificial ionospheric modification: the metal oxide space cloud experiment. Radio Sci 52(5):539-558. https://doi.org/10.1002/2016R S005988

Delamere PA, Swift DW, Stenbaek-Nielsen HC (2001) An explanation of the ion cloud morphology in the crres plasma injection experiments. J Geophys Res 106(A10):21289. https://doi.org/10.1029/2000ja000353

Drake JF, Mulbrandon M, Huba JD (1988) Three-dimensional equilibrium and stability of ionospheric plasma clouds. Phys Fluids 31(11):3412. https:// doi.org/10.1063/1.866906

Eliason L, Lundin R, Holmgren G (1988) Energetic electron enhancements due to the TOR chemical releases. Adv Space Res 8(1):93. https://doi. org/10.1016/0273-1177(88)90347-X

Foppl H, Haerendel G, Haser L, Loidl J, Lütjens P, Lüst R et al (1967) Artificial strontium and barium clouds in the upper atmosphere. Planet Space Sci 15(2):357-372. https://doi.org/10.1016/0032-0633(67)90200-0 
Gatsonis NA, Hastings DE (1991) A three-dimensional model and initial time numerical simulation for an artificial plasma cloud in the ionosphere. J Geophys Res Space Phys. https://doi.org/10.1029/90JA02249

Goldman SR, Baker L, Ossakow SL, Scannapieco AJ (1976) Striation formation associated with barium clouds in an inhomogeneous ionosphere. J Geophys Res 81(28):5097-5113. https://doi.org/10.1029/JA081i028p05097

Haerendel G, Lüst R, Rieger E (1967) Motion of artificial ion clouds in the up-per atmosphere. Planet Space Sci 15(1):1-18. https://doi.org/10.1016/00320633(67)90062-1

Holmgren G, Kintner PM, Kelley MC (1981) Artificial particle and wave stimulation in the trigger experiment. Adv Space Res 1(2):311. https://doi.org/10.1016/02731177(81)90305-7

Huba JD, Bernhardt PA, Lyon JG (1992) Preliminary study of the CRRES magnetospheric barium releases. J Geophys Res: Space Phys. https://doi. org/10.1029/91JA02144

Hu YG, Zhao ZY, Zhang YN (2012) Numerical simulation on the early dynamics of barium clouds released in the ionosphere. Acta Phys Sin 61(8):536-552. https://doi.org/10.1007/s11783-011-0280-z

Kelley MC, Livingston R (2003) Barium cloud striations revisited. J Geophys Res 108(A1):1044. https://doi.org/10.1029/2002JA009412

Lloyd KH, Haerendel G (1973) Numerical modeling of the drift and deformation of ionospheric plasma clouds and of their interaction with other layers of the ionosphere. J Geophys Res 78(31):7389-7415. https://doi. org/10.1029/ja078i031p07389

Ma TZ, Schunk RW (1991) Plasma cloud expansion in the ionosphere: three-dimensional simulation. J Geophys Res Space Phys. https://doi. org/10.1029/90JA02618

Ma TZ, Schunk RW (1993) Ionization and expansion of barium clouds in the ionosphere. J Geophys Res Space Phys. https://doi.org/10.1029/92JA0 1552

Ma TZ, Schunk RW (1994) Dynamics of three-dimensional plasma clouds with coupling to the background ionosphere. J Geophys Res 99(A4):6331. https://doi.org/10.1029/93ja02645

Ma TZ, Schunk RW (1990) A two-dimensional model of plasma expansion in the ionosphere. Planet Space Sci 38(6):723. https://doi.org/10.1016/00320633(90)90032-I

Mitchell HG, Fedder JA, Huba JD, Zalesak ST (1985) Transverse motion of highspeed barium clouds in the ionosphere. Space Phys J Geophys Res. https ://doi.org/10.1029/93ja02645

Morse DL, Destler WW (1973) Laboratory simulation of artificial plasma clouds in the ionosphere. J Geophys Res 78(31):7417-7430. https://doi. org/10.1029/ja078i031p07417

Oraevsky VN, Ruzhin YY, Badin VI, Deminov MG (2002) Alfven wave generation by means of high orbital injection of barium cloud in magnetosphere. Adv Space Res 29(9):1327-1334. https://doi.org/10.1016/S0273 $-1177(02) 00187-4$

Pavlov VA, Pinegin AN, Smirnovskii IR (1993) Plasma-perturbation evolution in the F-region and estimation of ionospheric parameters from sounding data. Radiophys Quantum Electron 36(3-4):131-138. https://doi. org/10.1007/BF01037199

Pressman J, Marrmo FF, Aschenbrand LM (1960) Artificial electron clouds-VI: Low altitude study, release of cesium at 69, 82 and $91 \mathrm{~km}$. Planet Space Sci 2(4):228

Rosenberg NW (1971) Observations of striation formation in a barium ion cloud. J Geophys Res 76(28):6856-6864. https://doi.org/10.1029/ja076 i028p06856
Rozhansky VA, Veselova IY, Voskoboynikov SP (1990) Three-dimensional computer simulation of plasma cloud evolution in the ionosphere. Planet Space Sci 38(11):1375-1386. https://doi.org/10.1016/0032-0633(90)90113 $-5$

Samir U, Wright KH, Stone NH (1983) The expansion of a plasma into a vacuum: Basic phenomena and processes and applications to space plasma physics. Rev Geophys 21(7):1631-1646. https://doi.org/10.1029/ RG021i007p01631

Scholer M (1970) On the motion of artificial ion clouds in the magnetosphere. Planetary Space Sci 18(7):977-1004. https://doi.org/10.1016/00320633(70)90101-7

Schunk RW, Szuszczewicz EP (1988) Early-time plasma expansion characteristics of ionized clouds in the ionosphere. J Geophys Res Space Phys 93(A11):12901-12915. https://doi.org/10.1029/JA093iA11 p12901

Schunk RW, Szuszczewicz EP (1991) Plasma expansion characteristics of ionized clouds in the ionosphere: macroscopic formulation. J Geophys Res Space Phys 96(A2):1337-1349. https://doi.org/10.1029/90JA02345

Valenzuela A, Haerendel G, Foppl H, Melzner F, Neuss H, Rieger E et al (1986) The AMPTE artificial comet experiments. Nature 320(6064):700-703. https //doi.org/10.1038/320700a0

Xie LH, Li L, Wang JD, Zhang YT (2014) Three-dimensional, two-species magneto-hydrodynamic studies of the early time behaviors of the combined release and radiation effects satellite $\mathrm{g} 2$ barium release. Phys Plasmas 21(4):042903. https://doi.org/10.1063/1.4871729

Xie LH, Li L, Wang JD, Tao R (2015) Determining wind field and electric field by a barium release experiment in the ionosphere. Sci China: Earth Sci 58(7):1210-1215. https://doi.org/10.1007/s11430-014-5051-9

Zakharov YP (2002) Laboratory simulation of artificial plasma releases in space. Adv Space Res 29(9):1335-1344. https://doi.org/10.1016/s0273 $-1177(02) 00184-9$

Zalesak ST, Drake JF, Huba JD (1988) Dynamics of three-dimensional ionospheric plasma clouds. Radio Sci 23(4):591-598. https://doi.org/10.1029/ rs023i004p00591

Zalesak ST, Drake JF, Huba JD (1990) Three-dimensional simulation study of ionospheric plasma clouds. Geophys Res Lett 17(10):1597-1600. https:// doi.org/10.1029/gl017i010p01597

Zhao HS, Feng J, Xu ZW, Wu J, Wu ZS, Xu B et al (2016) A temporal threedimensional simulation of samarium release in the ionosphere. J Geophys Res: Space Phys 121(10):10508-10519. https://doi. org/10.1002/2016JA022425

Zhang X, Sun A, Tian L, Zhang GJ (2019) Three-dimensional fluid simulations of the Cs plasma release in the ionosphere. AIP Adv 9(1):015117. https://doi. org/10.1063/1.5079433

\section{Publisher's Note}

Springer Nature remains neutral with regard to jurisdictional claims in published maps and institutional affiliations. 